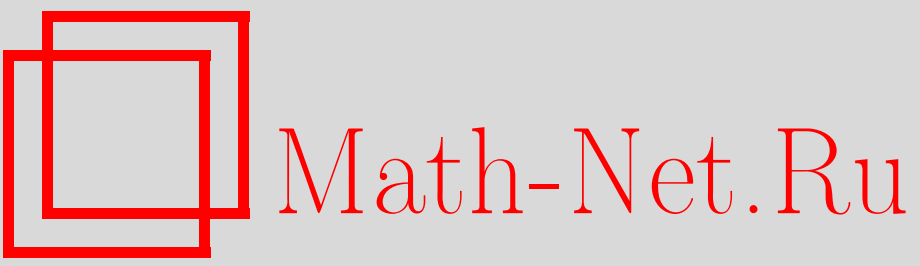

Н. М. Николов, Я. С. Станев, И. Т. Тодоров, Рациональная конформная теория поля в четырехмерном пространстве-времени, ТМФ, 2002, том 132, номер 2, 300317

DOI: https://doi.org/10.4213/tmf363

Использование Общероссийского математического портала Math-Net.Ru подразумевает, что вы прочитали и согласны с пользовательским соглашением http://www . mathnet.ru/rus/agreement

Параметры загрузки:

IP : 18.234 .156 .22

26 апреля 2023 г., $18: 13: 18$ 
ТЕОРЕТИЧЕСКАЯ

И МАТЕМАТИЧЕСКАЯ

ФИЗИКА

Том 132, № 2

август, 2002

(С) 2002 г. Н.М. Николов ${ }^{*}$, Я.С. Станев ${ }^{\dagger}$, И. Т. Тодоров

\title{
РАЦИОНАЛЬНАЯ КОНФОРМНАЯ ТЕОРИЯ ПОЛЯ В ЧЕТЫРЕХМЕРНОМ ПРОСТРАНСТВЕ-ВРЕМЕНИ
}

\begin{abstract}
Установленная недавно рациональность корреляционных функций в глобально конформно-инвариантной квантовой теории поля, удовлетворяющей аксиомам Вайтмана, используется для построения семейства решаемых моделей в четырехмерном пространстве-времени Минковского. Подробно рассматривается модель нейтрального скалярного поля $\phi$ размерности 2. Она зависит от положительного вещественного параметра $c$, аналога центрального заряда Вирасоро, и при всех (конечных) $c$ допускает бесконечное число сохраняющихся симметричных тензорных токов. Показано, что алгебра операторных произведений поля $\phi$ совпадает с более простой алгеброй, порожденной билокальным скалярным полем $V\left(x_{1}, x_{2}\right)$ размерности $1+1$. Моды $V$ вместе с единичным оператором порождают бесконечномерную алгебру Ли $\mathfrak{L}_{V}$, вакуумные (т.е. младшего веса с нулевой энергией) представления которой зависят только от центрального заряда $c$. Доказано, что вайтмановская положительность (т.е. унитарность представлений алгебры $\mathfrak{L}_{V}$ ) эквивалентна условию $c \in \mathbb{N}$.
\end{abstract}

Ключевые слова: аксиомы Вайтмана, операторное разложение, билокальные поля, представления бесконечномерных алгебр Ли.

\section{1. ВВЕДЕНИЕ}

Задача построения конформно-инвариантной модели квантовой теории поля с использованием одетых вертексных операторов и (глобальных) операторных произведений была сформулирована более 30 лет назад ([1]-[15], см. также в [16] обзор этих ранних работ и дальнейшие ссылки). Повторный всплеск интереса к данному предмету произошел (см., например, [17]-[23]) в результате появления двумерной конформной теории поля (KTП) (ныне вошедшей в учебники - см., например, [24], где можно найти библиографию оригинальных работ). Новый импульс был придан открытием АдС-КТП соответствия и связанным с этим интенсивным изучением $N=4$ суперсимметричной теории Янга-Миллса (см. [25], [26] и приведенную там библиографию).

\footnotetext{
*Institute for Nuclear Research and Nuclear Energy, Sofia, Bulgaria. E-mail: mitov@inrne.bas.bg

†Dipartimento di Fisica, Universita di Roma 'Tor Vergata', I. N.F.N.- Sezione di Roma 'Tor Vergata', Roma, Italy. E-mail: stanev@roma2.infn.it

${ }^{\ddagger}$ Erwin Schrödinger International Institute for Mathematical Physics, Wien, Austria. E-mail: todorov@inrne.bas.bg, itodorov@esi.ac.at
} 
Настолщая работа ${ }^{1)}$ мотивирована главным образом концепцией рациональной конформной теории поля (РКТП). Хотя это понятие возникло в рамках двумерной КТП, в недавней работе [28] высказано предположение, что оно может оказаться сушественным при любом числе измерений пространства-времени. Мы подробно рассматриваем приведенньй в [28] простейший выходящий за рамки свободных полей пример - модель нейтрального скалярного поля размерности 2. Более сложные (и потенциально более интересные) модели, включаюшие поля размерностей 3 и 4 , обсуждаются лишь кратко.

Начнем с напоминания основных результатов работы [28], которые позволяют вывести общие выражения для четырехточечных функций Вайтмана.

Добавляя к аксиомам Вайтмана условия глобальной конформной инвариантности (ГКИ) локальных наблюдаемых (т.е. условия инвариантности корреляционных функций по отношению к однозначному действию четырехкратного накрытия $G=S U(2,2)$ конформной групшы во всех случаях, когда $x$ и $g x$ (при $g \in G$ ) принадлежат пространству Минковского), мы приходим к принципу Гюйгенса: локальные поля $\phi(x), \psi(y)$ коммутируют, если разность $x-y$ не изотропна; более того,

$$
\left[(x-y)^{2}\right]^{N}[\phi(x), \psi(y)]=0 \text { при } N \gg 1
$$

(см. теорему 4.1 и предложение 4.3 в работе [28], где приведена точная граница для $N$ ). Этот результат основан на том факте, что пару пространственноподобно разделенных точек в пространстве Минковского можно подходящим конформным преобразованием отобразить во времениподобно разделенную пару. (Таким образом, условие ГКИ является более сильным требованием, чем инвариантность функций Швингера относительно действия евклидовой конформной группы.) Из принципа Гюйгенса (и из положительности энергии) следует, что распределения Вайтмана являются рашиональными функциями вида

$$
\mathcal{W}\left(x_{1}, \ldots, x_{n}\right) \equiv\langle 1, \ldots, n\rangle=P\left(x_{1}, \ldots, x_{n}\right) \prod_{1 \leqslant j<k \leqslant n}\left(\rho_{j k}\right)^{-\mu_{j k}},
$$

где $P$ - многочлен (вообще говоря, тензорнозначный),

$$
x_{j k} \equiv x_{j}-x_{k}, \quad \rho_{j k}=x_{j k}^{2}+i 0 x_{j k}^{0}, \quad x^{2}=\mathbf{x}^{2}-x_{0}^{2}, \quad \mu_{j k} \in \mathbb{Z}_{+}
$$

(см. теорему 3.1 в работе [28]; слагаемое $i 0 x_{j k}^{0}$, существенное только в том случае, когда $\rho_{j k}$ возникает в знаменателях, задает контур интегрирования для распределений Вайтмана, отвечаюший условию положительности энергии, см. [29], [30]). Положительность нормы в гильбертовом пространстве учитьвается при использовании операторных разложений и классификации унитарных неприводимых представлений $G$ с положительной энергией [31].

Продолжая обсуждение раздела 5 работы [28], выведем обший вид усеченной четырехточечной функции нейтрального скалярного поля $\phi$ целочисленной размерности $d$ при условии ГКИ.

\footnotetext{
1) См. подробное изложение в [27].
} 
Объединяя предложение 5.3 и следствие 4.4 из работы [28], можно записать усеченные четырехточечные функции $\mathcal{W}_{4}^{t}=\langle 1234\rangle-\langle 12\rangle\langle 34\rangle-\langle 13\rangle\langle 24\rangle-\langle 14\rangle\langle 23\rangle$ как

$$
\begin{gathered}
\mathcal{W}_{4}^{t}(d) \equiv \mathcal{W}^{t}\left(x_{1}, \ldots, x_{4} ; d\right)=\mathcal{D}_{d}\left(\rho_{i j}\right) \mathcal{P}_{d}\left(\eta_{1}, \eta_{2}\right), \\
\mathcal{D}_{d}\left(\rho_{i j}\right)=\frac{\left(\rho_{13} \rho_{24}\right)^{d-2}}{\left(\rho_{12} \rho_{23} \rho_{34} \rho_{14}\right)^{d-1}}, \quad \mathcal{P}_{d}\left(\eta_{1}, \eta_{2}\right)=\sum_{\substack{i, j \geqslant 0 \\
i+j \leqslant 2 d-3}} c_{i j} \eta_{1}^{i} \eta_{2}^{j},
\end{gathered}
$$

где $\eta_{i}-$ конформно-инвариантные отношения

$$
\eta_{1}=\frac{\rho_{12} \rho_{34}}{\rho_{13} \rho_{24}}, \quad \eta_{2}=\frac{\rho_{14} \rho_{23}}{\rho_{13} \rho_{24}} .
$$

При $x_{j k}^{2} \neq 0$ можно игнорировать член $i 0 x_{j k}^{0}$ в определении $\rho_{j k}(1.3)$. Кроссинг-симметрия, которая следует из локальности, приводит к инвариантности по отношению к группе перестановок $\mathcal{S}_{3}$ из шести элементов, порожденной операциями

$$
\begin{aligned}
& s_{12}: \mathcal{P}_{d}\left(\eta_{1}, \eta_{2}\right) \mapsto \eta_{2}^{2 d-3} \mathcal{P}_{d}\left(\frac{\eta_{1}}{\eta_{2}}, \frac{1}{\eta_{2}}\right)=\mathcal{P}_{d}\left(\eta_{1}, \eta_{2}\right), \\
& s_{23}: \mathcal{P}_{d}\left(\eta_{1}, \eta_{2}\right) \mapsto \eta_{1}^{2 d-3} \mathcal{P}_{d}\left(\frac{1}{\eta_{1}}, \frac{\eta_{2}}{\eta_{1}}\right)=\mathcal{P}_{d}\left(\eta_{1}, \eta_{2}\right)
\end{aligned}
$$

(включая также $s_{13}=s_{12} s_{23} s_{12}=s_{23} s_{12} s_{23}$, откуда $\left.\mathcal{P}_{d}\left(\eta_{2}, \eta_{1}\right)=\mathcal{P}_{d}\left(\eta_{1}, \eta_{2}\right)\right)$. В результате остаются следуюшие ${ }^{2)} \llbracket d^{2} / 3 \rrbracket$ независимых коэффициентов:

$$
\begin{gathered}
c_{i j} \text { при } i \leqslant j \leqslant \frac{2 d-3-i}{2}, \\
c_{i j}=c_{j i}=c_{i, 2 d-3-i-j}=c_{2 d-3-i-j, i}=c_{j, 2 d-3-i-j}=c_{2 d-3-i-j, j} .
\end{gathered}
$$

Настояшая статья посвящена главным образом обзору случая $d=2$, т.е. минимальному из тех значений $d$, для которых сушествует ненулевая усеченная четырехточечная функция $\mathcal{W}_{4}^{t}(d)$. Вводя сокрашенные обозначения, мы положим в этом случае

$$
\begin{gathered}
\langle 12\rangle=\frac{c}{2}(12)^{2}, \quad\langle 123\rangle=c(12)(23)(13), \\
\mathcal{W}_{4}^{t}(d=2)=c(12)(23)(34)(14)\left(1+\eta_{1}+\eta_{2}\right), \\
(i j)=\left(4 \pi^{2} \rho_{i j}\right)^{-1} .
\end{gathered}
$$

Параметр

$$
c=8 \frac{\langle 12\rangle\langle 23\rangle\langle 13\rangle}{(\langle 123\rangle)^{2}}
$$

инвариантен относительно изменения масштаба $\phi$. Он также определяет нормировку двухточечной функции тензора энергии-импульса и, таким образом, выглядит как обобщение центрального заряда Вирасоро.

Случаи $d>2$ (среди которых случай $d=4$ представляется особенно интересным) кратко обсуждаются в заключительном разделе 6 .

\footnotetext{
2) Здесь $\llbracket a \rrbracket$ означает целую часть $a\left(\llbracket d^{2} / 3 \rrbracket=1,3,5,8\right.$ при $\left.d=2,3,4,5\right)$.
} 
Работа построена следующим образом.

В разделе 2 с помошью экстраполяшии однопетлевого выражения для $\mathcal{W}_{4}^{t}$ на пяти- и шеститочечные функции выписывается операторное разложение двух полей $\phi$ в терминах билокального скалярного поля $V\left(x_{1}, x_{2}\right)$ размерности $1+1$, которое удовлетворяет (свободному) уравнению Д'Аламбера по каждому аргументу. Как следствие показано, что поле $V$ принадлежит алгебре операторных разложений, порожденной полем $\phi,-$ свойство, верное только в четырехмерном пространстве-времени. Из свободного полевого уравнения для $V$ следует, что усеченные $n$-точечные функции $\phi$ выражаются как суммы однопетлевых диаграмм с пропагаторами $(i j)$ и с общим множителем $c$ для всех $n \geqslant 3$.

В разделе 3 устанавливается сушествование бесконечного набора законов сохранения: член (12)(34), содержаший сингулярность на световом конусе, воспроизводится вкладом в операторное разложение произведения двух $\phi$ бесконечного числа сохраняющихся симметричных бесследовых тензорных токов четного ранга

$$
T_{2 l}(x, \zeta)=T_{\mu_{1} \ldots \mu_{2 l}}(x) \zeta^{\mu_{1}} \ldots \zeta^{\mu_{2 l}}, \quad \square_{\zeta} T_{2 l}(x, \zeta)=0=\frac{\partial^{2}}{\partial x_{\mu} \partial \zeta^{\mu}} T_{2 l}(x, \zeta)
$$

(при условии, что мы включаем также член $l=0$, полагая $T_{0}(x)=\phi(x)$ ). При положительных целых $c$ можно выразить $\phi$ как сумму нормальных произведений свободных полей:

$$
\phi(x)=\frac{1}{2} \sum_{i=1}^{N}: \varphi_{i}^{2}(x):, \quad\left\langle 0\left|\varphi_{i}\left(x_{1}\right) \varphi_{j}\left(x_{2}\right)\right| 0\right\rangle=\delta_{i j}(12) .
$$

Тензор энергии-импульса в этом случае также дается суммой свободно-полевых выражений:

$$
T_{2}(x, \zeta)=\sum_{i=1}^{c}:\left\{\left(\zeta \cdot \partial \varphi_{i}(x)\right)^{2}-\frac{1}{2} \zeta^{2} \partial_{\mu} \varphi_{i} \partial^{\mu} \varphi_{i}+\frac{1}{6}\left[\zeta^{2} \square-(\zeta \cdot \partial)^{2}\right] \varphi_{i}^{2}(x)\right\}: .
$$

Усеченные $n$-точечные функции поля $T_{2}(x, \zeta)$ остаются равными выражению для свободного безмассового скалярного поля при всех $c>0$. Таким образом, параметр $c$ действительно играет роль четырехмерного обобщения центрального заряда Вирасоро.

В разделе 4 исследуется разложение по модам билокального поля $V$, которое естественно возникает в так называемой аналитической компактной картине. Здесь представлена бесконечномерная алгебра Ли $\mathfrak{L}_{V}$, порожденная модами $V_{n m}\left(z_{1}, z_{2}\right)$ поля $V$ и единичньмм оператором.

В разделе 5 доказывается, что унитарные представления алгебры $\mathfrak{L}_{V}$ с положительной энергией соответствуют положительным целочисленным $c$. В случае выполнения условий из предложения 1 (см. ниже) тот же результат выводится для исходной полевой алгебры двумерного скалярного поля $\phi$. Отсюда следует, что $\phi$ принадлежит классу Борхерса системы свободных полей (более подробно о данной концепции см. [29], [30]).

В разделе 6 показано, как методы, предложенные в данной работе, применяются к киральному току размерности 3 и к плотности действия размерности 4 . 


\section{2. ОДНОПЕТЛЕВЫЕ $n$-ТОЧЕЧНЫЕ ФУНКЦИИ. ОПЕРАТОРНОЕ РАЗЛОЖЕНИЕ В ТЕРМИНАХ БИЛОКАЛЬНОГО ПОЛЯ}

Начнем с переписывания выражения для общей четырехточечной функции нейтрального скалярного поля $\phi(x)$ размерности 2, удовлетворяющего условию ГКИ (см. (1.8)), в форме, удобной для обобщения на $n$-точечную функцию. Согласно (1.8) имеем

$$
\langle 1234\rangle=\langle 12\rangle\langle 34\rangle+\langle 13\rangle\langle 24\rangle+\langle 14\rangle\langle 23\rangle+\mathcal{W}_{4}^{t}, \quad\langle i j\rangle=\frac{c}{2}(i j)^{2},
$$

где усеченную четырехточечную функцию Вайтмана можно записать как сумму вкладов трех ящичных диаграмм:

$$
\mathcal{W}_{4}^{t}=c\{(12)(34)(23)(14)+(12)(34)(13)(24)+(13)(24)(14)(23)\} .
$$

Уравнение (2.2) позволяет записать подобную симметричную сумму однопетлевых выражений для усеченной $n$-точечной функции:

$$
\begin{gathered}
\mathcal{W}_{n}^{t}\left(x_{1}, \ldots, x_{n}\right)=\frac{c}{2} \sum_{\sigma \in \operatorname{Perm}\{2 \ldots n\}}\left(1 \sigma_{2}\right) \sigma_{\sigma_{2} \sigma_{3} \ldots \sigma \sigma_{n-1} \sigma_{n}}\left(1 \sigma_{n}\right), \\
\sigma_{i} \sigma_{j}=\left\{\begin{array}{ll}
\left(\sigma_{i} \sigma_{j}\right), & \sigma_{i}<\sigma_{j}, \\
\left(\sigma_{j} \sigma_{i}\right), & \sigma_{j}<\sigma_{i},
\end{array} \quad n=2,3,4, \ldots\right.
\end{gathered}
$$

Более того, можно доказать

ПРЕДЛОЖЕНИЕ 1. Если представление (2.3) имеет место при $n \leqslant 4$, то предел

$$
V\left(x_{1}, x_{2}\right)=\lim _{\substack{\rho_{13} \rightarrow 0 \\ \rho_{23} \rightarrow 0}}(2 \pi)^{4} \rho_{13} \rho_{23}\left\{\phi\left(x_{1}\right) \phi\left(x_{2}\right) \phi\left(x_{3}\right)-\langle 13\rangle \phi\left(x_{2}\right)-\langle 23\rangle \phi\left(x_{1}\right)-\langle 123\rangle\right\}
$$

существует как билокальное поле (независимо от $x_{3}$ ). То же поле $V$ входит в операторное разложсение произведения двух $\phi:$

$$
\phi\left(x_{1}\right) \phi\left(x_{2}\right)=\langle 12\rangle+(12) V\left(x_{1}, x_{2}\right)+: \phi\left(x_{1}\right) \phi\left(x_{2}\right):,
$$

где каждое из трех слагаемых в правой части ортогонально двум другим. Поле $V\left(x_{1}, x_{2}\right)$ удовлетворяет свободному уравнению Д'Аламбера по каждому аргументу.

НАБРОСОК ДОКАЗАТЕЛЬСТВА. Из уравнения (2.2) и из условия сохранения тензора энергии-импульса следует, что представление (2.3) верно при $n \leqslant 6$. Однопетлевое выражение для шеститочечной функции позволяет вывести соотношение (2.4). Полученное из (2.5) выражение для корреляционной функции произведения двух $V$

$$
\left\langle 0\left|V\left(x_{1}, x_{2}\right) V\left(x_{3}, x_{4}\right)\right| 0\right\rangle=c\{(13)(24)+(14)(23)\}
$$

удовлетворяет уравнению Д'Аламбера по каждому аргументу. В силу локальной коммутативности и теоремы Ри-Шлидера [29], [30] операторное поле $V$ удовлетворяет этому уравнению во всей своей области определения. Подробное доказательство этого и последуюших результатов данного раздела будет опубликовано отдельно.

Из формулы (2.6) ясно, что поле $V\left(x_{1}, x_{2}\right)$ несингулярно при совпадаюших аргументах и, более того, его значение на диагонали воспроизводит исходное локальное поле:

$$
\phi(x)=\frac{1}{2} V(x, x) .
$$


ПРЕДЛОЖЕНИЕ 2. Пусть $\phi(x)$ - ГКИ поле Вайтмана размерности 2, удовлетворяющее условиям предложсения 1. Тогда усеченные п-точечные функиии ф имеют вид (2.3) при всех $n$.

НАБРОСОК ДОКАЗАТЕЛЬСТВА. Из уравнения (2.3) при $n \leqslant 6$ следует разложение вида

$$
\begin{aligned}
& \phi\left(x_{1}\right) \phi\left(x_{2}\right) \phi\left(x_{3}\right)|0\rangle=\langle 123\rangle|0\rangle+ \\
& \quad+\sum_{\substack{i=1,2,3 \\
j<k, j \neq i \neq k}}\left\{\langle j k\rangle \phi\left(x_{i}\right)+i j i k V\left(x_{j}, x_{k}\right)+(j k): V\left(x_{j}, x_{k}\right) \phi\left(x_{i}\right):\right\}|0\rangle+ \\
& \quad+: \phi\left(x_{1}\right) \phi\left(x_{2}\right) \phi\left(x_{3}\right):|0\rangle
\end{aligned}
$$

(здесь $i, j, k$ образуют перестановки $1,2,3)$. Отсюда следует утверждение предложения.

\section{3. РАЗЛОЖКНИЕ $V\left(x_{1}, x_{2}\right)$ ПО ЛОКАЛЬНЫМ \\ ПОЛЯМ. БЕСКОНЕЧНЫЙ НАБОР СОХРАНЯЮЩИХСЯ ТЕНЗОРНЫХ ТОКОВ}

Наша модель обладает бесконечным числом сохраняющихся локальных тензорных токов. Точнее говоря, билокальное поле $V\left(x_{1}, x_{2}\right)$ можно разложить в ряд по сохраняющимся симметричным бесследовым тензорным полям четного ранга $T_{2 l}(x, \zeta)(1.10)($ с твистом, равным разности размерности и ранга, т.е. 2):

$$
V\left(x_{1}, x_{2}\right)=2 \sum_{l=0}^{\infty} C_{l} K_{l}\left(x_{12} \cdot \partial_{2}, \rho_{12} \square_{2}\right) T_{2 l}\left(x_{2}, x_{12}\right),
$$

что воспроизводит четырехточечную функцию (2.6). Здесь

$$
K_{l}(s, t)=\frac{(2 l+1) !}{(l !)^{2}} \int_{0}^{1} d \alpha \alpha^{l}(1-\alpha)^{l} e^{\alpha s} \sum_{n=0}^{\infty} \frac{\left(-\frac{\alpha(1-\alpha)}{4} t\right)^{n}}{n !(2 l+1)_{n}}, \quad K_{l}(0,0)=1
$$

$\partial_{2}$ - производная по $x_{2}$ при фиксированном $x_{12},(\nu)_{n}=\Gamma(n+\nu) / \Gamma(\nu), \square_{2}-$ соответствующий оператор Д'Аламбера, который выбран для преобразования двухточечной функции $\left\langle 0\left|T_{2 l}\left(x_{2}, \zeta_{2}\right) T_{2 l}\left(x_{3}, \zeta_{3}\right)\right| 0\right\rangle$ в трехточечную:

$$
K_{l}\left(x_{12} \cdot \partial_{2}, \rho_{12} \square_{2}\right) \frac{\left(x_{12} \cdot r\left(x_{23}\right) \cdot \zeta\right)^{2 l}}{\rho_{23}^{2 l+2}}=\frac{(X \cdot \zeta)^{2 l}}{\rho_{13} \rho_{23}},
$$

где

$$
\xi \cdot r\left(x_{23}\right) \cdot \zeta=\xi \cdot \zeta-2 \frac{\left(\xi \cdot x_{23}\right)\left(\zeta \cdot x_{23}\right)}{\rho_{23}}, \quad X:=X_{12}^{3}:=\frac{x_{13}}{\rho_{13}}-\frac{x_{23}}{\rho_{23}}, \quad X^{2}=\frac{\rho_{12}}{\rho_{13} \rho_{23}}
$$

При проверке выражения (3.3) для $K_{l}$ вида (3.2) (см. [23]) используется соотношение

$$
\left(\frac{\square y}{4}\right)^{n} \frac{(y \cdot \zeta)^{m}}{\left(y^{2}\right)^{\nu}}=\frac{(\nu)_{n}(\nu-m-1)_{n}}{\left(y^{2}\right)^{n+\nu}}(y \cdot \zeta)^{m} \text { при } \zeta^{2}=0
$$

5 Теоретическая и математическая физика, т. 132, № 2, 2002 г. 
(при $\left.y=x_{23}+\alpha x_{12}\right)$. Для того чтобы вычислить индивидуальный вклад $T_{2 l}$ в четырехточечную функцию полей $\phi$, требуется трехточечная функция

$$
\left\langle 0\left|\phi\left(x_{1}\right) \phi\left(x_{2}\right) T_{2 l}\left(x_{3}, \zeta\right)\right| 0\right\rangle=2 N_{l} C_{l}\langle 12\rangle\left(X^{2}\right)^{l+1}\left(\zeta^{2}\right)^{l} C_{2 l}^{1}(\widehat{X} \cdot \hat{\zeta}), \quad \widehat{X}:=\frac{X}{\sqrt{X^{2}}}
$$

где $N_{l}>0, C_{n}^{1}(z)$ - многочлен Гегенбауэра, удовлетворяющий уравнению

$$
\left\{\left(1-z^{2}\right) \frac{d^{2}}{d z^{2}}-3 z \frac{d}{d z}+n(n+2)\right\} C_{n}^{1}(z)=0, \quad C_{n}^{1}(1)=n+1 .
$$

Записывая нормировочную константу в $(3.5)$ в виде произведения $N_{l} C_{l}$, мы пользуемся тем фактом, что трехточечная функция обрашается в нуль, когда структурная константа $C_{l}=0$.

Подставляя разложение (2.5) в четырехточечную функцию $(2.1),(2.2)$ и используя (2.6), ортогональность и разложение $(3.1)$ для $V\left(x_{3}, x_{4}\right)$, находим

$$
\begin{gathered}
\left\langle 0\left|\phi\left(x_{1}\right) \phi\left(x_{2}\right) V\left(x_{3}, x_{4}\right)\right| 0\right\rangle=c(12)((13)(24)+(14)(23))= \\
=2 \sum_{l=0}^{\infty} C_{l} K_{l}\left(x_{34} \cdot \partial_{4}, \rho_{34} \square_{4}\right)\left\langle 0\left|\phi\left(x_{1}\right) \phi\left(x_{2}\right) T_{2 l}\left(x_{4}, x_{34}\right)\right| 0\right\rangle= \\
=4\langle 12\rangle \sum_{l=0}^{\infty} N_{l} C_{l}^{2} \frac{(4 l+1) !}{(2 l) !^{2}} \times \\
\quad \times \int_{0}^{1} d \alpha \alpha^{2 l}(1-\alpha)^{2 l} \frac{\left(-\frac{\alpha(1-\alpha)}{4} \rho_{34} \square_{4}\right)^{n}}{n !(2 l+1)_{n}} \rho_{34}^{l}\left(X_{y}^{2}\right)^{l+1} C_{2 l}^{1}\left(\widehat{X}_{y} \cdot \hat{x}_{34}\right), \\
X_{y}=\frac{x_{1}-y}{\rho_{1 y}}-\frac{x_{2}-y}{\rho_{2 y}}, \quad y=x_{4}+\alpha x_{34}, \\
\rho_{i y}=\rho_{i 4}(1-\alpha)+\alpha \rho_{i 3}-\alpha(1-\alpha) \rho_{34}, \quad i=1,2 .
\end{gathered}
$$

Наряду со стандартными конформно-инвариантными отношениями $\eta_{1}$ и $\eta_{2}(1.5)$ введем разность $\epsilon=1-\eta_{2}$, которая стремится к нулю при $x_{34} \rightarrow 0$ (или $x_{12} \rightarrow 0$ ):

$$
\epsilon=1-\eta_{2}=O\left(x_{34}\right)=O\left(x_{12}\right) \text {. }
$$

ПРЕДЛОЖЕНИЕ 3. При

$$
N_{l} C_{l}^{2}=c\left(\begin{array}{l}
4 l \\
2 l
\end{array}\right)^{-1}
$$

вклад $V\left(x_{3}, x_{4}\right)$ в четырехточечную функцию (2.1) воспроизводится суперпозицией (3.7) трехточечных функций поля $T_{2 l}$ с твистом 2 ,

$$
\begin{aligned}
\frac{\left\langle 0\left|V\left(x_{1}, x_{2}\right) V\left(x_{3}, x_{4}\right)\right| 0\right\rangle}{(13)(24)} & =c\left(1+\frac{1}{1-\epsilon}\right)= \\
& =2 c \sum_{l=0}^{\infty}(4 l+1) \int_{0}^{1}\left[\frac{\epsilon \alpha(1-\alpha)}{1-\epsilon \alpha}\right]^{2 l} \frac{d \alpha}{1-\epsilon \alpha} .
\end{aligned}
$$


ДОКАЗАТЕЛЬСТВО этого утверждения приводится в приложении А работы [27].

Тождество Уорда-Такахаши для хронологически упорядоченной трехточечной функции тензора энергии-импульса позволяет вычислить нормировку $N_{1} C_{1}$ функции Вайтмана (3.5):

$$
\left\langle 0\left|\phi\left(x_{1}\right) \phi\left(x_{2}\right) T_{2}\left(x_{3}, \zeta\right)\right| 0\right\rangle=\frac{\langle 12\rangle}{3 \pi^{2}} X^{2}\left(X^{2} \zeta^{2}-4(X \cdot \zeta)^{2}\right),
$$

т.е.

$$
N_{1} C_{1}=-\frac{1}{3 \pi^{2}} .
$$

Сравнение этого результата с (3.9) показывает, что и $N_{1}$, и $C_{1}$ являются трансцендентными числами, и только комбинация $N_{1} C_{1}^{2}$ рациональна:

$$
C_{1}=-\frac{\pi^{2}}{2}, \quad N_{1}=\frac{2}{3 \pi^{4}}, \quad N_{1} C_{1}^{2}=\frac{1}{6} .
$$

ЗАмечание 1. Поучительно отметить, что вклад каждого $T_{2 l}$ в отношение (3.10) (определяемый $l$-м членом в правой части) содержит логарифмическую функцию от $1-\epsilon$, тогда как бесконечная сумма является рациональной функцией $\epsilon$.

\section{4. БЕСКОНЕЧНОМЕРНАЯ АЛГЕБРА ЛИ ПОЛЕВЫХ МОД И ЕЕ БИЛОКАЛЬНАЯ РЕАЛИЗАЦИЯ}

Конформная компактификация $\bar{M}=\mathbb{S}^{3} \times \mathbb{S}^{1} / \mathbb{Z}_{2}$ пространства Минковского $M=\mathbb{R}^{3,1}$ приводит к естественному понятию конформной энергии - генератора (изометрических) врашений времениподобной окружности $\mathbb{S}^{1}$ и соответствуюшего дискретного базиса полевых мод. Следуя [17], мы параметризуем $\bar{M}$ с помошью комплексных координат $z=\left(z_{a}, a=1,2,3,4\right)$, фиксированных инволюцией $z \mapsto z^{*}:=\bar{z} / \bar{z}^{2}$ :

$$
\bar{M}=\left\{z=\left(z_{a} \in \mathbb{C}, a=1, \ldots, 4\right) ; \quad z_{a}^{*}:=\frac{\bar{z}_{a}}{\bar{z}^{2}}=z_{a} \quad\left(z^{2}=\sum_{a} z_{a}^{2}=: \mathbf{z}^{2}+z_{4}^{2}\right)\right\} .
$$

Из этого условия следует свойство

$$
z^{2} \bar{z}^{2}=1, \quad \frac{z_{a} z_{b}}{z^{2}}=\bar{z}_{a} z_{b}=z_{a} \bar{z}_{b} \in \mathbb{R} \text { при } z \in \bar{M},
$$

которое, в свою очередь, характеризует данную параметризацию $\bar{M}$. Мы выберем отображение вложения $M \subset \bar{M}$ в виде

$$
M \ni\left(x^{0}, \mathbf{x}\right) \mapsto \mathbf{z}=\omega^{-1}(x) \mathbf{x}, \quad z_{4}=\frac{1-x^{2}}{2 \omega(x)}, \quad \omega(x)=\frac{1+x^{2}}{2}-i x^{0} .
$$

Ясно, что значение $z$, определяемое из (4.3), удовлетворяет условиям (4.2); в частности,

$$
z^{2}=\frac{\overline{\omega(x)}}{\omega(x)}=\frac{\left(1+i x^{0}\right)^{2}+\mathbf{x}^{2}}{\left(1-i x^{0}\right)^{2}+\mathbf{x}^{2}}=\frac{1}{\bar{z}^{2}}, \quad\left|z^{2}\right|=z \cdot \bar{z}=1 \text { при } z \in \bar{M} .
$$


Конус на бесконечности $K_{\infty}=\bar{M} \backslash M$ состоит из тех $z \in \bar{M}$, для которых конформный множитель $\omega_{z}^{-1}\left(\omega_{z}:=\omega(x(z))\right)$ обрашается в нуль:

$$
z \in K_{\infty} \text { тогда и только тогда, когда } 2 \omega_{z}^{-1}=\left(1+z_{4}\right)^{2}+\mathbf{z}^{2}=0 .
$$

Воспользуемся тем фактом, что плоская метрика на $\bar{M}$ связана с пуанкаре-инвариантной метрикой на $M$ комплексным конформным множителем $\omega(4.3)$ :

$$
d z^{2}=\mathbf{d z}^{2}+d z_{4}^{2}=\omega^{-2}(x) d x^{2}, \quad d x^{2}=\mathbf{d x}^{2}-d x_{0}^{2} .
$$

Скалярному полю $\phi_{M}(x)$ размерности $d$ в пространстве Минковского мы поставим в соответствие аналитическое поле $\phi(z)$ в z-картине, определяемое как

$$
\phi(z)=(2 \pi)^{d} \omega_{z}^{d} \phi_{M}(x(z)), \quad \omega_{z}=\frac{2}{\left(1+z_{4}\right)^{2}+\mathbf{z}^{2}}=\omega(x(z)) .
$$

Термин аналитическое поле оправдан тем обстоятельством, что из положительности энергии следует аналитичность векторнозначной функции $\phi(z)|0\rangle$ при $|z|^{2}<1$. Действительно, труба будушего $T_{+}=\left\{\zeta \in \mathbb{C}^{4} ; \operatorname{Im} \zeta^{0}>|\operatorname{Im} \underline{\zeta}|\right\}$ - область аналитичности $\phi_{M}(\zeta)|0\rangle$ (см. [29], [30]) - отображается в комплексную окрестность $\mathfrak{T}_{+}$четырехмерного единичного шара $\mathbb{B}^{4} ;$ более точно, имеем

$$
\begin{aligned}
\mathbb{B}^{4} & =\left\{\xi \in \mathbb{R}^{4} ; \quad \xi^{2}:=\underline{\xi}^{2}+\xi_{4}^{2}<1\right\}, \\
\mathbb{B}^{4} \times \mathbb{S}^{1} / \mathbb{Z}_{2} & =\left\{z=\xi e^{i \tau} ; \quad \xi \in \mathbb{B}^{4}, \quad \tau \in \mathbb{R}\right\} \subset \mathfrak{T}_{+} .
\end{aligned}
$$

Отметим, что $\bar{M}$ возникает как граница пятимерного многообразия $\mathbb{B}^{4} \times \mathbb{S}^{1} / \mathbb{Z}^{2}$ :

$z \in \bar{M}$ тогда и только тогда, когда $z=e^{i \tau} \hat{z}, \quad \tau \in \mathbb{R}, \quad \hat{z} \in \mathbb{S}^{3}=\left\{\hat{z} \in \mathbb{R}^{4} ; \quad \hat{z}^{2}=1\right\}$.

Конформный гамильтониан $H$ в этой картине является не чем иным, как (эрмитовым) генератором трансляций по $\tau$ :

$$
e^{i H t} \phi(z) e^{-i H t}=e^{i t d} \phi\left(e^{i t} z\right) \quad \text { или }[H, \phi(z)]=\left(d+z_{a} \frac{\partial}{\partial z_{a}}\right) \phi(z), \quad H|0\rangle=0 .
$$

Разложение $\phi$ по собственным модам $H$ имеет вид

$$
\phi(z)=\sum_{n \in \mathbb{Z}} \phi_{n}(z), \quad\left[\phi_{n}(z), H\right]=n \phi_{n}(z)
$$

$\operatorname{Moдъl~} \phi_{n}(z)$ можно записать как степенные рядыпо $z_{a}$ и $1 / z^{2}$, являющиеся однородными по $z$ степени $-n-d$. 
Для свободного поля $\varphi(z)$ размерности $d=1$ моды $\varphi_{ \pm n}$ являются однородными гармоническими многочленами, порождающими пространство размерности $n^{2}-$ пространство $S O(4)$-симметричных бесследовых тензоров ранга $n-1:\left(\begin{array}{c}n+2 \\ 3\end{array}\right)-\left(\begin{array}{c}n \\ 3\end{array}\right)=n^{2}$; в частности, $\varphi_{0}(z)=0, \varphi_{1}(z)=a_{1} / z^{2}, \varphi_{-1}(z)=a_{-1}, \varphi_{2}(z)=a_{2}^{\mu} z_{\mu} /\left(z^{2}\right)^{2}, \varphi_{-2}(z)=a_{-2}^{\mu} z_{\mu}$ и т.д. Они подчинены каноническим коммутационным соотношениям [17]

$$
\begin{gathered}
{\left[\varphi_{n}(z), \varphi_{m}(w)\right]=\frac{\left(w^{2}\right)^{\frac{n-1}{2}}}{\left(z^{2}\right)^{\frac{n+1}{2}}} C_{|n|-1}^{1}(\hat{z} \cdot \widehat{w}) \epsilon(n) \delta_{n,-m}, \quad z=\sqrt{z^{2}} \hat{z},} \\
\epsilon(n)=\left\{\begin{array}{lll}
1 & \text { при } n>0, \\
0 & \text { при } n=0, \\
-1 & \text { при } n<0 .
\end{array}\right.
\end{gathered}
$$

(Здесь используется тот факт, что двухточечная функция $\langle 0|\varphi(z) \varphi(w)| 0\rangle=1 /(z-w)^{2}$ возникает как производящая функция для многочленов Гегенбауэра, определенных в (3.6).)

Моды билокального поля $V$ ведут себя как произведения $\varphi$-мод:

$$
\begin{gathered}
\Delta_{z} V_{n m}(z, w)=0=\Delta_{w} V_{n m}(z, w) \\
\left(z \cdot \frac{\partial}{\partial z}+n+1\right) V_{n m}(z, w)=0=\left(w \cdot \frac{\partial}{\partial w}+m+1\right) V_{n m}(z, w) \\
V=\sum_{\substack{n, m \\
n \neq 0, m \neq 0}} V_{n m}
\end{gathered}
$$

(условие однородности согласуется с уравнением Лапласа, только если положить $V_{0 m}=$ $\left.0=V_{n 0}\right)$. Моды $d=2$ поля $\phi$ наиболее удобным образом выражаются как бесконечные суммы $V$-мод:

$$
2 \phi_{n}(z)=\sum_{\nu \in \mathbb{Z}} V_{\nu, n-\nu}(z, z), \quad V_{m n}(z, z)=V_{n m}(z, z) .
$$

Компоненты $V_{\nu, n-\nu}(z, z)$ мод $\phi_{n}(z)$ (в отличие от соответствующих компонент для $\left.\varphi_{n}(z)\right)$ порождают бесконечномерное пространство. Особенно замечательным является тот факт, что пространство состояний для заданной энергии $n$ всегда конечномерно. Это следует из аналитичности векторнозначной функции $V_{n m}(z, w)|0\rangle$ при $\operatorname{Im} z>0$, $\operatorname{Im} w>0$. Действительно, из (4.8) и (4.13) следует, что

$$
V_{n m}(z, w)|0\rangle=0 \text {, если } n \geqslant 0 \text { или } m \geqslant 0 .
$$

Поэтому только $n-1$ членов бесконечной суммы (4.14) дают вклад в вектор $\phi_{-n}(z)|0\rangle$ :

$$
2 \phi_{-n}(z)|0\rangle=\sum_{\nu=1}^{n-1} V_{-\nu, \nu-n}(z, z)|0\rangle .
$$

Заметим, что согласно (2.5) вакуумное операторное разложение остается в силе в $z$-картине, если положить

$$
(12)=\frac{1}{z_{12}^{2}} \Rightarrow\langle 12\rangle=\frac{c}{2}(12)^{2}=\frac{c}{2}\left(z_{12}^{2}\right)^{-2}, \quad z^{2}=\mathbf{z}^{2}+z_{4}^{2}
$$


Моды поля $\phi$ не порождают (бесконечномерную) алгебру Ли: кроме того, коммутатор $\left[\phi\left(z_{1}\right), \phi\left(z_{2}\right)\right]$ включает все сохраняющиеся тензоры $T_{2 l}\left(z_{2}, z_{12}\right)$ с твистом 2 (и их производные по первому аргументу). Вместе с единичным оператором тензоры $T_{2 l}$ $(l=0,1, \ldots)$ в действительности исчерпывают сингулярные члены в операторном разложении $\phi(z) \phi(w)|0\rangle$. Возникающая при этом коммутаторная алгебра радикально упрошается для коллинеарных $z_{j}=\zeta_{j} e\left(e^{2}=1\right)$; она сводится в этом случае к алгебре Вирасоро:

$$
\left[L_{n}, L_{m}\right]=(n-m) L_{n+m}+\frac{c}{12} n\left(n^{2}-1\right) \delta_{n,-m} \quad \text { при } \phi_{n}(\zeta e)=\frac{L_{n}}{\zeta^{n+2}}, \quad L_{n}=\phi_{n}(e) .
$$

Дело в том, что в одномерном случае второй аргумент $z_{12}$ тензора $T_{2}\left(z_{2}, z_{12}\right)$ сокрашает сингулярный множитель $1 / z_{12}^{2}$ в операторном разложении.

В отличие от $\phi_{n}(z)$, моды $V_{n m}$ поля $V$, удовлетворяюшие (4.13), и единичный оператор сами по себе порождают некоторую алгебру Ли $\mathfrak{L}_{V}$ (которая является центральным расширением вешественной бесконечномерной симплектической алгебры Ли):

$$
\begin{aligned}
& {\left[V_{n_{1} n_{2}}\left(z_{1}, z_{2}\right), V_{n_{3} n_{4}}\left(z_{3}, z_{4}\right)\right]=} \\
&=c \prod_{j=1}^{4}\left(z_{j}^{2}\right)^{-\frac{n_{j}+1}{2}}\left\{C_{\left|n_{1}\right|-1}^{1}\left(\hat{z}_{1} \cdot \hat{z}_{3}\right) C_{\left|n_{2}\right|-1}^{1}\left(\hat{z}_{2} \cdot \hat{z}_{4}\right) \delta_{n_{1},-n_{3}} \delta_{n_{2},-n_{4}}+\right. \\
&\left.+C_{\left|n_{1}\right|-1}^{1}\left(\hat{z}_{1} \cdot \hat{z}_{4}\right) C_{\left|n_{2}\right|-1}^{1}\left(\hat{z}_{2} \cdot \hat{z}_{3}\right) \delta_{n_{1},-n_{4}} \delta_{n_{2},-n_{3}}\right\} \epsilon\left(n_{1}\right) \epsilon\left(n_{2}\right)+ \\
&+\left(z_{1}^{2}\right)^{-\frac{n_{1}+1}{2}}\left(z_{3}^{2}\right)^{-\frac{n_{3}+1}{2}} C_{\left|n_{1}\right|-1}^{1}\left(\hat{z}_{1} \cdot \hat{z}_{3}\right) \epsilon\left(n_{1}\right) \delta_{n_{1},-n_{3}} V_{n_{2} n_{4}}\left(z_{2}, z_{4}\right)+ \\
&+\left(z_{2}^{2}\right)^{-\frac{n_{2}+1}{2}}\left(z_{3}^{2}\right)^{-\frac{n_{3}+1}{2}} C_{\left|n_{2}\right|-1}^{1}\left(\hat{z}_{2} \cdot \hat{z}_{3}\right) \epsilon\left(n_{2}\right) \delta_{n_{2},-n_{3}} V_{n_{1} n_{4}}\left(z_{1}, z_{4}\right)+ \\
&+\left(z_{1}^{2}\right)^{-\frac{n_{1}+1}{2}}\left(z_{4}^{2}\right)^{-\frac{n_{4}+1}{2}} C_{\left|n_{1}\right|-1}^{1}\left(\hat{z}_{1} \cdot \hat{z}_{4}\right) \epsilon\left(n_{1}\right) \delta_{n_{1},-n_{4}} V_{n_{2} n_{3}}\left(z_{2}, z_{3}\right)+ \\
&+\left(z_{2}^{2}\right)^{-\frac{n_{2}+1}{2}}\left(z_{4}^{2}\right)^{-\frac{n_{4}+1}{2}} C_{\left|n_{2}\right|-1}^{1}\left(\hat{z}_{2} \cdot \hat{z}_{4}\right) \epsilon\left(n_{2}\right) \delta_{n_{2},-n_{4}} V_{n_{1} n_{3}}\left(z_{1}, z_{3}\right) .
\end{aligned}
$$

Согласно (4.14) $\phi$-моды принадлежат этой алгебре. Вакуумное представление алгебры $\mathfrak{L}_{V}$ характеризуется условием положительности энергии (4.15).

\section{5. УНИТАРНЫЕ ВАКУУМНЫЕ ПРЕДСТАВЛЕНИЯ АЛГЕБРЫ $\mathfrak{L}_{V}$}

Введем сначала антиинволюцию в $\mathfrak{L}_{V}$ и соответствуюшее внутреннее произведение в $\mathcal{H}$. Определим звездочка-оператор в алгебре мод, полагая

$$
V_{n m}(z, w)^{*}=V_{-m,-n}(w, z)=V_{-n,-m}(z, w) \text { при } z, w \in \bar{M},
$$

так что $V(z, w)^{*}=V(z, w)$.

ЗАмечАниЕ 2. Антиинволюция (5.1) включает соответствие между однородными гармоническими функшиями степени $n-1$ и $-n-1$. Если при $n, m>0$ записать

$$
V_{-n,-m}(z, w)=V_{-n,-m}^{b_{1} \ldots b_{n-1}, a_{1} \ldots a_{m-1}} z_{b_{1}} \ldots z_{b_{n-1}} w_{a_{1}} \ldots w_{a_{m-1}},
$$


то получим

$$
V_{-n,-m}(z, w)^{*}=V_{m n}(w, z)=\frac{1}{w^{2} z^{2}} V_{m n}^{a_{1} \ldots a_{m-1}, b_{1} \ldots b_{n-1}} \frac{w_{a_{1}}}{w^{2}} \ldots \frac{w_{a_{m-1}}}{w^{2}} \frac{z_{b_{1}}}{z^{2}} \ldots \frac{z_{b_{n-1}}}{z^{2}},
$$

где и $V_{-n,-m}$, и $V_{n m}$ являются симметричными бесследовыми тензорами ранга $(n-$ $1, m-1)$ (по отношению к индексам $a_{i}$ и $b_{j}$ по отдельности).

Будем называть представление алгебры $\mathfrak{L}_{V}$ в гильбертовом пространстве $\mathcal{H}$ унитарHbl.M, если (положительное) скалярное произведение в $\mathcal{H}$ и сопряжение $(5.1)$ в $\mathfrak{L}_{V}$ связаны как

$$
(\Phi, X \Psi)=\left(X^{*} \Phi, \Psi\right) \text { для каждого } X \in \mathfrak{L}_{V}, \quad \Phi, \Psi \in \mathcal{H}^{F},
$$

где $\mathcal{H}^{F}$ - плотное подпространство векторов с конечной энергией из $\mathcal{H}$, которые принадлежат области определения любого $X$ в $\mathfrak{L}_{V}$.

$\mathrm{B} \mathcal{H}_{V}$ можно ввести (не обязательно положительное) внутреннее произведение $\langle\cdot, \cdot\rangle$, удовлетворяющее условию (5.2), определив бра-вакуум с помощью условий, сопряженHыx (4.15),

$$
\langle 0| V_{n m}=0 \text { во всех случаях, кроме } n>0 \text { и } m>0,
$$

и полагая $\langle 0 \mid 0\rangle=1$. Основным результатом данного раздела является следующая характеризация унитарного вакуумного представления алгебры $\mathfrak{L}_{V}$.

Теорема. Внутреннее произведение в $\mathcal{H}_{V}$, определенное для (нормированного) вакуумного вектора, удовлетворяющего (4.15) и (5.3), и для $V_{n m}(z, w)$, подчиняющегося условию (4.18), является положительно-полуопределенным тогда и только тогда, когда $c \in \mathbb{Z}_{+}=\{0,1,2, \ldots\}$.

ДокАЗАТЕЛЬСтво. Зафиксируем единичный вектор $e \in \mathbb{S}^{3}$ и рассмотрим одномерную подалгебру $\mathfrak{L}_{V}^{e}$ в $\mathfrak{L}_{V}$, порожденную операторами

$$
v_{n m}:=V_{n m}(e, e) \in \mathfrak{L}_{V}^{e} \subset \mathfrak{L}_{V}, \quad n, m \in \mathbb{Z}, \quad e^{2}=1 .
$$

Из (4.18) следует, что операторы $v_{n m}$ удовлетворяют коммутационным соотношениям мод одномерного (кирального) билокального тока:

$$
\begin{aligned}
& {\left[v_{n_{1} m_{1}}, v_{n_{2} m_{2}}\right]=c n_{1} m_{1}\left(\delta_{n_{1},-n_{2}} \delta_{m_{1},-m_{2}}+\delta_{n_{1},-m_{2}} \delta_{m_{1},-n_{2}}\right)+} \\
& \quad+n_{1}\left(\delta_{n_{1},-n_{2}} v_{m_{1} m_{2}}+\delta_{n_{1},-m_{2}} v_{m_{1} n_{2}}\right)+m_{1}\left(\delta_{m_{1},-n_{2}} v_{n_{1} m_{2}}+\delta_{m_{1},-m_{2}} v_{n_{1} n_{2}}\right)
\end{aligned}
$$

ЛЕммА. Существует вектор $\left|\Delta_{n}\right\rangle \in \mathcal{H}_{V}^{(n(n+1))}$, норма которого кратна $c(c-$ 1) ... $(c-n+1)$ :

$$
\begin{gathered}
\left\langle\Delta_{n}\right|=\frac{1}{n !}\left\langle 0|| \begin{array}{cccc}
v_{11} & v_{12} & \ldots & v_{1 n} \\
v_{21} & v_{22} & \ldots & v_{2 n} \\
\ldots & \ldots & \ldots & \ldots \\
v_{n 1} & v_{n 2} & \ldots & v_{n n}
\end{array}\right|, \\
\left\langle\Delta_{n} \mid \Delta_{n}\right\rangle \equiv \|\left|\Delta_{n}\right\rangle \|^{2}=(n+1) ! c(c-1) \ldots(c-n+1) .
\end{gathered}
$$


ДокАЗАТЕЛЬСтво. Из (5.5) следует, что квадрат нормы многочлена степени $n$ относительно переменной $v_{k l}$ является многочленом степени (не превосходяшей) $n$ по $c$. Покажем, что $\left\langle\Delta_{n} \mid \Delta_{n}\right\rangle$ обрашается в нуль для целых $c$ в интервале $0 \leqslant c<n$. Для этого заметим, что если $c$ - положительное целое число, а $\vec{J}_{m}, m \in \mathbb{Z},-c$-мерные операторнозначные векторы $\vec{J}_{m}=\left\{J_{m}^{i}, i=1, \ldots, c\right\}$, удовлетворяющие равенствам

$$
\left[J_{m}^{i}, J_{n}^{j}\right]=m \delta_{m,-n} \delta_{i j}, \quad m, n \in \mathbb{Z}, \quad i, j=1, \ldots, c
$$

то нормальные произведения

$$
v_{l m}^{(c)}=: \vec{J}_{l} \cdot \vec{J}_{m}: \equiv \sum_{i=1}^{c}: J_{l}^{i} J_{m}^{i}:
$$

удовлетворяют коммутационным соотношениям (4.18). Если $c<n$, то появляющаяся в определении $\left\langle\Delta_{n}\right|$ величина $\left.\operatorname{det}\left(v_{i j}\right)\right|_{i, j=1, \ldots, n}$, являюшаяся определителем Грама скалярных произведений $n$ векторов в $c$-мерном пространстве, должна обрашаться в нуль. Коэффициент $(n+1)$ ! при старшей $(n-\ddot{и})$ степени $c$ вычисляется как сумма квадратов норм членов, входящих в разложение определителя.

Из приведенной леммы следует, что всегда, кроме случая, когда $c$ - положительное целое число, существуют векторы с отрицательной нормой. Для доказательства того, что при $c \in \mathbb{N}$ вакуумное представление $\mathfrak{L}_{V}$ действительно является унитарным, достаточно заметить, что в этом случае $V$ можно записать в виде

$$
V\left(z_{1}, z_{2}\right)=\sum_{i=1}^{c}: \varphi_{i}\left(z_{1}\right) \varphi_{i}\left(z_{2}\right):
$$

где $\varphi_{i}$ - взаимно коммутируюшие свободные поля нулевой массы, и вспомнить, что система свободных полей удовлетворяет всем аксиомам Вайтмана (включая положительность).

Попутно (в качестве прямого следствия леммы) мы установили следуюший результат.

ПРЕДЛОЖЕНИЕ 4. ВакуУмное представление двумерного билокального кирального поля

$$
v(z, w)=\frac{1}{z w} \sum_{n, m \in \mathbb{Z}} v_{n m} z^{-n} w^{-m}, \quad z, w \in \mathbb{C},
$$

модъ которого удовлетворяют коммутационным соотношениям (5.5) (и условию $v_{m n}|0\rangle=0$ во всех случаях, кроме $m<0$ и $\left.n<0\right)$, является унитарным только при положстельных цельх с.

Это аналог теоремы Каца-Радула [32] об унитарных представлениях $W_{1+\infty}$-алгебры. Ясно, что алгебра двумерного тензора энергии-импульса

$$
T(z)=\frac{1}{2} v(z, z)=\sum_{n \in \mathbb{Z}} L_{n} z^{-n-2},
$$


т.е. алгебра Вирасоро (4.17), является собственной подалгеброй в $\mathfrak{L}_{V}$, поскольку она допускает унитарные представления при всех $c \geqslant 1$, а также при дискретных сериях $c=c_{n}=1-6 /((n+1)(n+2))\left(n=1,2, \ldots\right.$, причем унитарный модуль Вирасоро $\mathcal{H}_{c_{n}}$ является факторпространством соответствуюшего модуля младшего веса по сингулярному вектору на уровне энергии (собственное значение оператора $\left.L_{0}\right) n(n+1)$ ).

Другая ситуация имеет место при $d=4$, поскольку в этом случае $V(z, w)$ гармонически зависит от каждого аргумента. Можно показать [27], что алгебра, включаюшая $V$, не превосходит исходную алгебру операторных разложений $\phi$, так что для нее справедлива приведенная выше теорема.

СлЕДствиЕ. В случае выполнения условий предложения 1 из теоремы следует, что квантовая теория поля ф с усеченной п-точечной функцией (2.3) удовлетворяет условию положительности Вайтмана тогда и только тогда, когда с натуральное число (в әтом случае ф принадлежит классу Борхерса набора свободньих полей).

\section{6. ОБОБЩЕНИЕ РЕЗУЛЬТАТОВ. ЗАКЛЮЧИТЕЛЬНЫЕ ЗАМЕЧАНИЯ}

Представленные выше результаты и методы применимы к полям высших размерностей и произвольной тензорной структуры. Мы установили [27] важные специальные случаи следуюшей гипотезы.

ГипотеЗА. Если нейтральное тензорное поле челочисленной размерности имеет усеченные п-точечные функиии, которые являются кратными соответствующим корреляторам нормальных произведений (производных) свободных полей при $n \leqslant 6$, то из условия положительности Вайтмана следует, что константа пропорииональности является положительным челым числом.

Как указано в разделе 2, для $d=2$ это утверждение следует из выражения для четырехточечной функции.

ПримеР 1. Рассмотрим сохраняюшийся ток, для которого первые пять усеченных корреляционных функций получены из соответствуюших корреляционных функций для тока в системе двухкомпонентых спиноров

$$
J^{\mu}\left(x ; c_{\psi}\right)=\sum_{j=1}^{c_{\psi}}: \psi_{j}^{*}(x) \widetilde{\sigma}^{\mu} \psi_{j}(x):, \quad-\widetilde{\sigma}^{0}=\widetilde{\sigma}_{0}=\mathbf{1}=\sigma_{0}, \quad \tilde{\sigma}^{j}=-\sigma^{j}=-\sigma_{j},
$$

путем замены положительного целого $c_{\psi}$ на произвольное вещественное число. Здесь $\psi_{j}$ - взаимно антикоммутируюшие свободные поля Вейля, для которых

$$
\left\langle 0\left|\psi_{j}\left(x_{1}\right) \psi_{k}^{*}\left(x_{2}\right)\right| 0\right\rangle=\delta_{j k} S\left(x_{12}\right), \quad S\left(x_{12}\right)=i \underset{\sim}{\partial_{2}}(12)=i \frac{x_{12}}{2 \pi^{2} \rho_{12}^{2}},
$$

где использованы обозначения

$$
\underset{\sim}{\partial_{2}}=\sigma_{\mu} \frac{\partial}{\partial x_{2 \mu}}, \quad \underset{\sim}{x}=\sigma_{\mu} x^{\mu}, \quad \sigma^{\mu} \tilde{\sigma}_{\nu}+\sigma_{\nu} \tilde{\sigma}^{\mu}=-2 \delta_{\nu}^{\mu} .
$$


Вводя спин-тензорные компоненты тока

$$
J(x)=J_{\alpha \dot{\beta}}(x)=\frac{1}{2} \sigma_{\mu} J^{\mu}=\sum_{j=1}^{c_{\psi}}: \psi_{j \alpha}(x) \psi_{j \dot{\beta}}^{*}(x):
$$

можно записать

$$
\begin{gathered}
J_{\alpha_{1} \dot{\beta_{1}}}\left(x_{1}\right) J_{\alpha_{2} \dot{\beta_{2}}}\left(x_{2}\right)-\left\langle 0\left|J_{\alpha_{1} \dot{\beta_{1}}}\left(x_{1}\right) J_{\alpha_{2} \dot{\beta_{2}}}\left(x_{2}\right)\right| 0\right\rangle=S_{\dot{\beta}_{1} \alpha_{2}}^{\mathrm{t}}\left(x_{12}\right) V_{\alpha_{1} \dot{\beta_{2}}}\left(x_{1}, x_{2}\right)+ \\
+S_{\alpha_{1} \dot{\beta_{2}}}\left(x_{12}\right) V_{\dot{\beta_{1} \alpha_{2}}}^{\mathrm{t}}\left(x_{1}, x_{2}\right)+: J_{\alpha_{1} \dot{\beta_{1}}}\left(x_{1}\right) J_{\alpha_{2} \dot{\beta_{2}}}\left(x_{2}\right):
\end{gathered}
$$

где $S^{\mathrm{t}}\left(V^{\mathrm{t}}\right)$ - транспонирование $S(V)$. Умножая обе части на $\left(2 \pi^{2} / i\right) \rho_{12} \tilde{x}_{12}^{\dot{\beta}_{2} \alpha_{2}}$ и полагая

$$
W_{\alpha_{1} \dot{\beta_{1}}}\left(x_{1}, x_{2}\right)=\frac{2 \pi^{2}}{i} \rho_{12} \tilde{x}_{12}^{\dot{\beta}_{2} \alpha_{2}}\left\{J_{\alpha_{1} \dot{\beta_{1}}}\left(x_{1}\right) J_{\alpha_{2} \dot{\beta}_{2}}\left(x_{2}\right)-\left\langle 0\left|J_{\alpha_{1} \dot{\beta_{1}}}\left(x_{1}\right) J_{\alpha_{2} \dot{\beta}_{2}}\left(x_{2}\right)\right| 0\right\rangle\right\}
$$

находим

$$
W_{\alpha_{1} \dot{\beta_{1}}}\left(x_{1}, x_{2}\right)=V_{\alpha_{1} \dot{\beta_{1}}}\left(x_{1}, x_{2}\right)+V_{\dot{\beta}_{1} \alpha_{1}}^{\mathrm{t}}\left(x_{1}, x_{2}\right)+: J_{\alpha_{1} \dot{\beta_{1}}}\left(x_{1}\right) \operatorname{tr}\left(\tilde{x}_{12} J\left(x_{2}\right)\right): \text {, }
$$

где билокальное поле $V$ удовлетворяет равенству

$$
\left\langle 0\left|V_{\alpha_{1} \dot{\beta_{1}}}\left(x_{1}, x_{2}\right) V_{\alpha_{2} \dot{\beta_{2}}}\left(x_{3}, x_{4}\right)\right| 0\right\rangle=S_{\alpha_{1} \dot{\beta_{2}}}\left(x_{14}\right) S_{\dot{\beta}_{1} \alpha_{2}}^{\mathrm{t}}\left(x_{23}\right),
$$

откуда следует, что

$$
\tilde{\partial}_{1}^{\dot{\alpha} \alpha_{1}} V_{\alpha_{1} \dot{\beta}_{1}}\left(x_{1}, x_{2}\right)=0=V_{\alpha_{1} \dot{\beta}_{1}}\left(x_{1}, x_{2}\right) \frac{\overleftarrow{\partial}}{\partial x_{2 \mu}} \tilde{\sigma}_{\mu}^{\dot{\beta}_{1} \beta}
$$

В результате $V_{\alpha \dot{\beta}}$ и нормальное произведение $J$ из правой части (6.7) можно определить по отдельности и, как и в разделе 5 , можно доказать, что $c_{\psi}\left(c_{\psi}-1\right) \ldots\left(c_{\psi}-n+1\right) \geqslant 0$ при $n=1,2, \ldots$.

ПРИмеР 2. Рассмотрим плотность лагранжиана

$$
\mathcal{L}_{F}(x)=-\frac{1}{4} \sum_{a=1}^{c_{F}}: F_{\mu \nu}^{a}(x) F_{a}^{\mu \nu}(x):, \quad c_{F} \in \mathbb{N},
$$

и соответствуюшее аналитическое продолжение усеченных функций Вайтмана на произвольные вещественные положительные $c_{F}$. Усеченная $n$-точечная функция полей $\mathcal{L}_{F}$ снова может быть записана как сумма $(n-1) ! / 2$ однопетлевых графов, причем пропагатор, соответствующий линии, соединяющей вершины 1 и 2 , равен

$$
\begin{aligned}
\mathcal{D}_{\lambda_{1} \mu_{1} \lambda_{2} \mu_{2}}\left(x_{12}\right) & =\frac{1}{4}\left\{\partial_{\lambda_{1}}\left(\partial_{\lambda_{2}} \eta_{\mu_{1} \mu_{2}}-\partial_{\mu_{2}} \eta_{\mu_{1} \lambda_{2}}\right)-\partial_{\mu_{1}}\left(\partial_{\lambda_{2}} \eta_{\lambda_{1} \mu_{2}}-\partial_{\mu_{2}} \eta_{\lambda_{1} \lambda_{2}}\right)\right\} \frac{1}{4 \pi^{2} \rho_{12}}= \\
& =\frac{r_{\lambda_{1} \lambda_{2}}\left(x_{12}\right) r_{\mu_{1} \mu_{2}}\left(x_{12}\right)-r_{\lambda_{1} \mu_{2}}\left(x_{12}\right) r_{\mu_{1} \lambda_{2}}\left(x_{12}\right)}{4 \pi^{2} \rho_{12}^{2}} .
\end{aligned}
$$


Это выражение для пропагатора входит также в операторное разложение произведения двух $\mathcal{L}$ (вместе с тензорнозначным билокальным полем),

$$
\begin{aligned}
& \langle 0| \mathcal{L}_{F}\left(x_{1}\right) \mathcal{L}_{F}\left(x_{2}\right)=\langle 0|\left\{2 c_{F} \mathcal{D}^{\lambda_{1} \mu_{1} \lambda_{2} \mu_{2}}\left(x_{12}\right) \mathcal{D}_{\lambda_{1} \mu_{1} \lambda_{2} \mu_{2}}\left(x_{12}\right)+\right. \\
& \left.+\mathcal{D}^{\lambda_{1} \mu_{1} \lambda_{2} \mu_{2}}\left(x_{12}\right) V_{\lambda_{1} \mu_{1} \lambda_{2} \mu_{2}}\left(x_{1}, x_{2}\right)+: \mathcal{L}_{F}\left(x_{1}\right) \mathcal{L}_{F}\left(x_{2}\right):\right\}, \\
& 2 \mathcal{D}^{\lambda_{1} \mu_{1} \lambda_{2} \mu_{2}} \mathcal{D}_{\lambda_{1} \mu_{1} \lambda_{2} \mu_{2}}=\frac{3}{\left(\pi \rho_{12}\right)^{4}} \text {. }
\end{aligned}
$$

При $c \in \mathbb{N}$ существует реализация $V$ как суммы нормальных произведений свободных максвелловских полей:

$$
V_{\lambda_{1} \mu_{1} \lambda_{2} \mu_{2}}\left(x_{1}, x_{2}\right)=: F_{\lambda_{1} \mu_{1}}^{a}\left(x_{1}\right) F_{\lambda_{2} \mu_{2}}^{a}\left(x_{2}\right): \text {. }
$$

Операторное разложение (6.12) позволяет вычислить усеченную четырехточечную функцию полей $\mathcal{L}_{F}$, которая возникает как специальный случай 5 -параметрического выражения для $\left.\mathcal{W}_{4}^{t}(d)\right|_{d=4}$, вычисленного исходя из уравнений (1.4)-(1.7):

$$
\begin{aligned}
\mathcal{W}_{4}^{t}(4)= & \frac{\rho_{13}^{2} \rho_{24}^{2}}{\rho_{12}^{3} \rho_{23}^{3} \rho_{34}^{3} \rho_{14}^{3}}\left\{c_{0}\left(1+\eta_{1}^{5}+\eta_{2}^{5}\right)+c_{1}\left(\eta_{1}+\eta_{2}+\eta_{1}^{4}+\eta_{2}^{4}+\eta_{1} \eta_{2}\left(\eta_{1}^{3}+\eta_{2}^{3}\right)\right)+\right. \\
& +c_{2}\left(\eta_{1}^{2}+\eta_{2}^{2}+\eta_{1}^{3}+\eta_{2}^{3}+\eta_{1}^{2} \eta_{2}^{2}\left(\eta_{1}+\eta_{2}\right)\right)+ \\
& \left.+b_{1} \eta_{1} \eta_{2}\left(1+\eta_{1}^{2}+\eta_{2}^{2}\right)+b_{2} \eta_{1} \eta_{2}\left(\eta_{1} \eta_{2}+\eta_{1}+\eta_{2}\right)\right\}
\end{aligned}
$$

здесь $c_{i} \equiv c_{0 i}$ при $i=0,1,2 ; b_{i} \equiv c_{1 i}$ при $i=1,2$. Действительно, вклад ящичной диаграммы (вычисленньй с использованием формул для следов степеней произведений $r_{\nu}^{\mu}$ из приложения В работы [27]) имеет вид

$$
\begin{aligned}
& \mathcal{W}_{\square}=c_{F} \mathcal{D}_{\mu_{1} \mu_{2}}^{\lambda_{1} \lambda_{2}}\left(x_{12}\right) \mathcal{D}_{\lambda_{1} \lambda_{4}}^{\mu_{1} \mu_{4}}\left(x_{14}\right) \mathcal{D}_{\lambda_{2} \lambda_{3}}^{\mu_{2} \mu_{3}}\left(x_{23}\right) \mathcal{D}_{\mu_{3} \mu_{4}}^{\lambda_{3} \lambda_{4}}\left(x_{34}\right)= \\
& =32 c_{F} \frac{1}{(2 \pi)^{4}\left(\rho_{12} \rho_{23} \rho_{34} \rho_{14}\right)^{2}}\left(1+\frac{\eta_{1}}{\eta_{2}}+\frac{\eta_{2}}{\eta_{1}}-\frac{2}{\eta_{2}}-\frac{2}{\eta_{1}}+\frac{1}{\eta_{1} \eta_{2}}\right),
\end{aligned}
$$

он входит в выражение для усеченной четырехточечной функции полей $\mathcal{L}(x)$

$$
\begin{aligned}
\mathcal{W}_{4}^{t} & =\left(1+s_{12}+s_{23}\right) \mathcal{W}_{\square}\left(x_{1}, x_{2}, x_{3}, x_{4}\right)= \\
& =\mathcal{W}_{\square}\left(x_{1}, x_{2}, x_{3}, x_{4}\right)+\mathcal{W}_{\square}\left(x_{2}, x_{1}, x_{3}, x_{4}\right)+\mathcal{W}_{\square}\left(x_{1}, x_{3}, x_{2}, x_{4}\right)
\end{aligned}
$$

и отвечает выражению (6.14) при $c_{0}=c_{2}=b_{1}=-c_{1} / 2=c_{F} / 8 \pi^{8}, b_{2}=0$. Первым локальным полем в разложении $V$ вокруг диагонали является тензор энергии-импульса

$$
T_{\nu}^{\mu}=\frac{1}{4} V_{\kappa \lambda}^{\kappa \lambda}(x, x) \delta_{\nu}^{\mu}-V_{\lambda \nu}^{\lambda \mu}(x, x)=-\mathcal{L}(x) \delta_{\nu}^{\mu}-V_{\lambda \nu}^{\lambda \mu}(x, x) .
$$

Обратно, билокальное тензорное поле

$$
V_{\lambda_{2} \mu_{2}}^{\lambda_{1} \mu_{1}}\left(x_{1}, x_{2}\right)=-V_{\lambda_{2} \mu_{2}}^{\mu_{1} \lambda_{1}}\left(x_{1}, x_{2}\right)=-V_{\mu_{2} \lambda_{2}}^{\lambda_{1} \mu_{1}}\left(x_{1}, x_{2}\right)
$$

появляется в операторном разложении произведения двух $T_{\nu}^{\mu}$ и определяется из последнего в два этапа. Во-первых, выводится формула

$$
\begin{aligned}
\left\langle 0\left|V_{\lambda_{2} \mu_{2}}^{\lambda_{1} \mu_{1}}\left(x_{1}, x_{2}\right) V_{\lambda_{4} \mu_{4}}^{\lambda_{3} \mu_{3}}\left(x_{3}, x_{4}\right)\right| 0\right\rangle= & c_{F} \mathcal{D}^{\lambda_{1} \mu_{1} \lambda_{3} \mu_{3}}\left(x_{13}\right) \mathcal{D}_{\lambda_{2} \mu_{2} \lambda_{4} \mu_{4}}\left(x_{24}\right)+ \\
& +c_{F} \mathcal{D}_{\lambda_{4} \mu_{4}}^{\lambda_{1} \mu_{1}}\left(x_{14}\right) \mathcal{D}_{\lambda_{2} \mu_{2}}^{\lambda_{3} \mu_{3}}\left(x_{23}\right)
\end{aligned}
$$

и из нее можно получить, что $V_{\lambda_{2} \mu_{2}}^{\lambda_{1} \mu_{1}}\left(x_{1}, x_{2}\right)$ удовлетворяет свободным уравнениям Максвелла по каждому аргументу. Во-вторых, этот факт используется для выделения вклада $V$ в операторное разложение произведения двух $T$. Условие положительности Вайтмана снова приводит к тому, что $c_{F} \in \mathbb{N}$. 
ЗАМЕчАнИЕ 3. Использование различных обозначений $c=c_{\phi}, \quad c_{\psi}$ и $c_{F}$ для констант, на которые умножаются усеченные функции нормальных произведений соответствующих свободных полей $\phi, \psi$ и $F_{\mu \nu}$, оправдано тем, что они соответствуют различным тензорным структурам (и исчерпывают их) в общей конформно-инвариантной трехточечной функции тензора энергии-импульса [19].

В то же время четырехточечные функции сохраняющегося тока $J_{\mu}$ и $\mathcal{L}(x)$ включают структуры, которые нельзя свести к нормальным произведениям свободных полей. Если, например, $b_{2} \neq 0$ в (6.14), то трехточечная функция полей $\mathcal{L}(x)$ не обрашается в нуль (в отличие от случая суперпозиции типа (6.10) нормальных произведений свободных максвелловских полей). Более обшим образом, имеется 4-параметрическое семейство допустимых четырехточечных функций поля $\mathcal{L}(x)$, ограниченных только условиями $c_{2}=-c_{0}-c_{1} \neq 2 c_{0}$, которые возникают из требования, чтобы никакое двумерное поле не появлялось в операторном разложении $\mathcal{L}\left(x_{1}\right) \mathcal{L}\left(x_{2}\right)$ (и чтобы тензор энергии-импульса был представлен в этом операторном разложении). Они согласованы только с трехточечными функциями для $T$ вида (6.17) (т.е. с третьей из трех допустимых структур в этой трехточечной функции, приведенной в [19], ср. замечание 3 ).

Подводя итог, отметим, что в поисках четырехмерной РКТП за пределами класса Борхерса свободных полей мы исключили теорию скалярного поля размерности 2 и пришли в итоге к следуюшей задаче.

Предположим, что единственными локальными полями в наблюдаемой алгебре, удовлетворяюшими ГКИ и имеюшими размерность $d \leqslant 4$, являются (сохраняюшийся бесследовый) тензор энергии-импульса $T_{\mu \nu}(x)$ и скалярное поле $\mathcal{L}(x)$ размерности 4 (играюшее роль плотности действия). Задача состоит в построении алгебры операторных разложений, согласованной с $n$-точечными функциями этих полей при $n \leqslant 4$, что позволит вычислить высшие корреляционные функции и реализовать условие положительности Вайтмана. Этот пример привлекателен, поскольку размерности базисных полей $\mathcal{L}_{\text {и }} T_{\mu \nu}$ жестко фиксированы. Кроме того, в любой перенормируемой квантовой теории поля можно определить (калибровочно-инвариантную) локальную плотность действия и тензор энергии-импульса.

Благодарности. Мы благодарим за поддержку и гостеприимство Erwin Schrödinger International Institute for Mathematical Physics (ESI) в Вене, где была выполнена основная часть данной работы. Мы с благодарностью отмечаем обсуждения с Хансом Борхерсом, Харалдом Гроссе, Якобом Ингвасоном и Юрием Неретиным. Работа Я.С. была частично поддержана EEC грантами HPRN-CT-2000-00122 и HPRN-CT-200000148 и грантом INTAS 99-1-590. Работа Н.Н. и И.Т. частично поддержана Bulgarian National Council for Scientific Research (грант F-828).

\section{Список литературы}

[1] А. М. Поляков. Письма в ЖЭТФ. 1970. Т. 12. С. 538.

[2] A. A. Migdal. Phys. Lett. B. 1971. V. 37. P. 386.

[3] G. Parisi, L. Peliti. Lett. Nuovo Cimento. 1971. V. 2. P. 627.

[4] E. Schreier. Phys. Rev. D. 1971. V. 3. P. 980.

[5] B. Schroer. Lett. Nuovo Cimento. 1971. V. 2. P. 867; Bjorken scaling and scale invariant quantum field theories. In: Scale and Conformal Symmetry in Hadron Physics. Ed. R. Gatto. 
New York: Wiley, 1973. P. 43.

[6] G. Mack, K. Symanzik. Commun. Math. Phys. 1972. V. 27. P. 247.

[7] K. Symanzik. Lett. Nuovo Cimento. 1972. V. 3. P. 734.

[8] S. Ferrara, R. Gatto, A. Grillo, G. Parisi. Phys. Lett. B. 1972. V. 38. P. 333; Lett. Nuovo Cimento. 1972. V. 4. P. 115; General consequences of conformal algebra. In: Scale and Conformal Symmetry in Hadron Physics. Ed. R. Gatto. New York: Wiley, 1973. P. 59.

[9] I. T. Todorov. Conformal invariant quantum field theory. In: Strong Interaction Physics. Lect. Notes in Phys. V. 17. Eds. W. Ruhl, A. Vancura. Berlin: Springer, 1972. P. 270.

[10] G. Mack, I. T. Todorov. Phys. Rev. D. 1973. V. 8. P. 1764.

[11] G. Mack. Conformal invariant quantum field theory. In: Scale and Conformal Symmetry in Hadron Physics. Ed. R. Gatto. New York: Wiley, 1973. P. 109; Renormalization and Invariance in Quantum Field Theory. New York: Plenum Press, 1974.

[12] А. М. Поляков. ЖЭТФ. 1974. Т. 66. С. 23.

[13] B. Schroer, J. A. Swieca, A. H. Volkel. Phys. Rev. D. 1975. V. 11. P. 1509.

[14] V. K. Dobrev, V. B. Petkova, S. G. Petrova, I. T. Todorov. Phys. Rev. D. 1976. V. 13. P. 887.

[15] G. Mack. Commun. Math. Phys. 1977. V. 53. P. 155.

[16] I. T. Todorov, M. C. Mintchev, V. B. Petkova. Conformal Invariance in Quantum Field Theory. Pisa: Scuola Normale Superiore, 1978.

[17] I. T. Todorov. Infinite dimensional Lie algebras in conformal QFT models. In: Conformal Groups and Related Symmetries. Physical Results and Mathematical Background. Lect. Notes in Phys. V. 261. Eds. A. O. Barut, H.-D. Doebner. Berlin: Springer, 1986. P. 387.

[18] J. Cardy. Nucl. Phys. B. 1987. V. 290. P. 355.

[19] Ya. S. Stanev. Bulg. J. Phys. 1988. V. 15. P. 93.

[20] K. Lang, W. Ruhl. Nucl. Phys. B. 1993. V. 402. P. 573.

[21] H. Osborn, A. Petkou. Ann. Phys. 1994. V. 231. P. 311.

[22] E. S. Fradkin, M. Ya. Palchik. Phys. Rep. 1998. V. 300. P. 1; Conformal Quantum Field Theory in $D$-dimensions. Dordrecht: Kluwer, 1996.

[23] F. A. Dolan, H. Osborn. Nucl. Phys. B. 2001. V. 599. P. 459; hep-th/0011040.

[24] P. Di Francesco, P. Mathieu, D. Senechal. Conformal Field Theories. Berlin: Springer, 1996.

[25] G. Aratyunov, B. Eden, A. C. Petkou, E. Sokatchev. Exceptional non-renormalization properties and OPE analysis of chiral 4-point functions in $N=4 \mathrm{SYM}_{4}$. hep-th/0103230.

[26] M. Bianchi, S. Kovacs, G. Rossi, Ya. S. Stanev. Properties of Konishi multiplet in $N=4$ SYM theory. hep-th/0104016.

[27] N. M. Nikolov, Ya. S. Stanev, I. T. Todorov. Four dimensional CFT models with rational correlation functions. hep-th/0110230; J. Phys. A (to be published).

[28] N. M. Nikolov, I. T. Todorov. Commun. Math. Phys. 2001. V. 218. P. 417; hep-th/0009004.

[29] P. Стритер, A. Вайтман. РСТ, спин и статистика и все такое. М.: Наука, 1966.

[30] Н. Н. Боголюбов, А. А. Логунов, А. И. Оксак, И. Т. Тодоров. Общие принципы квантовой теории поля. М.: Наука, 1987; Н. Н. Боголюбов, А. А. Логунов, И. Т. Тодоров. Основы аксиоматического подхода в квантовой теории поля. М.: Наука, 1969.

[31] G. Mack. Commun. Math. Phys. 1977. V. 55. P. 1.

[32] V. G. Kac, A. Radul. Commun. Math. Phys. 1993. V. 157. P. 125; Transform. Groups. 1996. V. 1. № 1-2. P. 41. 